\title{
Supplementary information: Electroosmotic flow in polarizable charged cylindrical nanopores
}

Rogério K. Bombardelli, ${ }^{*}$ Igor M. Telles, ${ }^{*}$ Alexandre P. dos Santos, ${ }^{*}$ and Yan Levin*

Instituto de Física, Universidade Federal do Rio Grande do Sul, Caixa Postal 15051, CEP 91501-970, Porto Alegre, RS, Brazil

E-mail: rogerio.bombardelli@ufrgs.br; igor.morais@ufrgs.br; alexandre.pereira@ufrgs.br; levin@if.ufrgs.br

${ }^{*}$ To whom correspondence should be addressed 


\section{Derivation of the polarization force}

Here, we derive the polarization force acting on a particle $i$. The force can be obtained from the derivatives of electrostatic energy calculated in third section, Electrostatic energy.

$$
\begin{array}{r}
\beta l_{B} F_{i}^{l}=-\frac{l_{B}^{2}}{L}\left[8 \sum _ { n = 1 } ^ { + \infty } \sum _ { m = 1 } ^ { + \infty } \gamma _ { m n } \left(f_{1} \frac{\partial f_{1}}{\partial x_{i}^{l}}+f_{2} \frac{\partial f_{2}}{\partial x_{i}^{l}}+\right.\right. \\
\left.f_{3} \frac{\partial f_{3}}{\partial x_{i}^{l}}+f_{4} \frac{\partial f_{4}}{\partial x_{i}^{l}}\right)+ \\
4 \sum_{n=1}^{+\infty} \gamma_{0 n}\left(g_{1} \frac{\partial g_{1}}{\partial x_{i}^{l}}+g_{2} \frac{\partial g_{2}}{\partial x_{i}^{l}}\right)+ \\
\left.\sum_{m=1}^{+\infty} \frac{2}{m} \frac{\left(\varepsilon_{w}-\varepsilon_{o}\right)}{\left(\varepsilon_{w}+\varepsilon_{o}\right)}\left(h_{1} \frac{\partial h_{1}}{\partial x_{i}^{l}}+h_{2} \frac{\partial h_{2}}{\partial x_{i}^{l}}\right)\right],
\end{array}
$$

where $F_{i}^{l}$ is $l$ cartesian component of the force acting on the particle $i$. With this notation $x_{i}^{(1)}=x_{i}, x_{i}^{(2)}=y_{i}$ and $x_{i}^{(3)}=z_{i}$.

$$
\frac{\partial}{\partial x_{i}^{l}}=\frac{\partial \rho_{i}}{\partial x_{i}^{l}} \frac{\partial}{\partial \rho_{i}}+\frac{\partial \theta_{i}}{\partial x_{i}^{l}} \frac{\partial}{\partial \theta_{i}}+\frac{\partial z_{i}}{\partial x_{i}^{l}} \frac{\partial}{\partial z_{i}} .
$$

The partial derivatives are

$$
\begin{gathered}
\frac{\partial \rho_{i}}{\partial x_{i}}=\frac{\partial \sqrt{x_{i}^{2}+y_{i}^{2}}}{\partial x_{i}}=\frac{x_{i}}{\sqrt{x_{i}^{2}+y_{i}^{2}}} \\
\frac{\partial \rho_{i}}{\partial y_{i}}=\frac{\partial \sqrt{x_{i}^{2}+y_{i}^{2}}}{\partial y_{i}}=\frac{y_{i}}{\sqrt{x_{i}^{2}+y_{i}^{2}}} \\
\frac{\partial \theta_{i}}{\partial x_{i}}=\frac{\partial \arctan \left(y_{i} / x_{i}\right)}{\partial x_{i}}=-\frac{y_{i}}{x_{i}^{2}+y_{i}^{2}} \\
\frac{\partial \theta_{i}}{\partial y_{i}}=\frac{\partial \arctan \left(y_{i} / x_{i}\right)}{\partial y_{i}}=\frac{x_{i}}{x_{i}^{2}+y_{i}^{2}}
\end{gathered}
$$


The $x$-derivatives are:

$$
\begin{aligned}
& \frac{\partial f_{1}}{\partial x_{i}}=\frac{q_{i} x_{i} k_{n}}{2 \rho_{i}}\left[I_{m-1}\left(k_{n} \rho_{i}\right)+I_{m+1}\left(k_{n} \rho_{i}\right)\right] \cos \left[m \theta_{i}\right] \cos \left[k_{n} z_{i}\right]+ \\
& \frac{q_{i} y_{i} m}{\rho_{i}^{2}} I_{m}\left(k_{n} \rho_{i}\right) \sin \left[m \theta_{i}\right] \cos \left[k_{n} z_{i}\right] \\
& \frac{\partial f_{2}}{\partial x_{i}}=\frac{q_{i} x_{i} k_{n}}{2 \rho_{i}}\left[I_{m-1}\left(k_{n} \rho_{i}\right)+I_{m+1}\left(k_{n} \rho_{i}\right)\right] \cos \left[m \theta_{i}\right] \sin \left[k_{n} z_{i}\right]+ \\
& \frac{q_{i} y_{i} m}{\rho_{i}^{2}} I_{m}\left(k_{n} \rho_{i}\right) \sin \left[m \theta_{i}\right] \sin \left[k_{n} z_{i}\right] \\
& \frac{\partial f_{3}}{\partial x_{i}}=\frac{q_{i} x_{i} k_{n}}{2 \rho_{i}}\left[I_{m-1}\left(k_{n} \rho_{i}\right)+I_{m+1}\left(k_{n} \rho_{i}\right)\right] \sin \left[m \theta_{i}\right] \cos \left[k_{n} z_{i}\right]- \\
& \frac{q_{i} y_{i} m}{\rho_{i}^{2}} I_{m}\left(k_{n} \rho_{i}\right) \cos \left[m \theta_{i}\right] \cos \left[k_{n} z_{i}\right] \\
& \frac{\partial f_{4}}{\partial x_{i}}=\frac{q_{i} x_{i} k_{n}}{2 \rho_{i}}\left[I_{m-1}\left(k_{n} \rho_{i}\right)+I_{m+1}\left(k_{n} \rho_{i}\right)\right] \sin \left[m \theta_{i}\right] \sin \left[k_{n} z_{i}\right]- \\
& \frac{q_{i} y_{i} m}{\rho_{i}^{2}} I_{m}\left(k_{n} \rho_{i}\right) \cos \left[m \theta_{i}\right] \sin \left[k_{n} z_{i}\right] \\
& \frac{\partial g_{1}}{\partial x_{i}}=\frac{q_{i} x_{i} k_{n}}{2 \rho_{i}}\left[I_{-1}\left(k_{n} \rho_{i}\right)+I_{1}\left(k_{n} \rho_{i}\right)\right] \cos \left[k_{n} z_{i}\right] \\
& \frac{\partial g_{2}}{\partial x_{i}}=\frac{q_{i} x_{i} k_{n}}{2 \rho_{i}}\left[I_{-1}\left(k_{n} \rho_{i}\right)+I_{1}\left(k_{n} \rho_{i}\right)\right] \sin \left[k_{n} z_{i}\right] \\
& \frac{\partial h_{1}}{\partial x_{i}}=\frac{q_{i} x_{i} m}{R \rho_{i}}\left(\frac{\rho_{i}}{R}\right)^{m-1} \cos \left[m \theta_{i}\right]+\frac{q_{i} y_{i} m}{\rho_{i}^{2}}\left(\frac{\rho_{i}}{R}\right)^{m} \sin \left[m \theta_{i}\right] \\
& \frac{\partial h_{2}}{\partial x_{i}}=\frac{q_{i} x_{i} m}{R \rho_{i}}\left(\frac{\rho_{i}}{R}\right)^{m-1} \sin \left[m \theta_{i}\right]-\frac{q_{i} y_{i} m}{\rho_{i}^{2}}\left(\frac{\rho_{i}}{R}\right)^{m} \cos \left[m \theta_{i}\right] \text {. }
\end{aligned}
$$


The $y$-derivatives are:

$$
\begin{aligned}
& \frac{\partial f_{1}}{\partial y_{i}}=\frac{q_{i} y_{i} k_{n}}{2 \rho_{i}}\left[I_{m-1}\left(k_{n} \rho_{i}\right)+I_{m+1}\left(k_{n} \rho_{i}\right)\right] \cos \left[m \theta_{i}\right] \cos \left[k_{n} z_{i}\right]- \\
& \frac{q_{i} x_{i} m}{\rho_{i}^{2}} I_{m}\left(k_{n} \rho_{i}\right) \sin \left[m \theta_{i}\right] \cos \left[k_{n} z_{i}\right] \\
& \frac{\partial f_{2}}{\partial y_{i}}=\frac{q_{i} y_{i} k_{n}}{2 \rho_{i}}\left[I_{m-1}\left(k_{n} \rho_{i}\right)+I_{m+1}\left(k_{n} \rho_{i}\right)\right] \cos \left[m \theta_{i}\right] \sin \left[k_{n} z_{i}\right]- \\
& \frac{q_{i} x_{i} m}{\rho_{i}^{2}} I_{m}\left(k_{n} \rho_{i}\right) \sin \left[m \theta_{i}\right] \sin \left[k_{n} z_{i}\right] \\
& \frac{\partial f_{3}}{\partial y_{i}}=\frac{q_{i} y_{i} k_{n}}{2 \rho_{i}}\left[I_{m-1}\left(k_{n} \rho_{i}\right)+I_{m+1}\left(k_{n} \rho_{i}\right)\right] \sin \left[m \theta_{i}\right] \cos \left[k_{n} z_{i}\right]+ \\
& \frac{q_{i} x_{i} m}{\rho_{i}^{2}} I_{m}\left(k_{n} \rho_{i}\right) \cos \left[m \theta_{i}\right] \cos \left[k_{n} z_{i}\right] \\
& \frac{\partial f_{4}}{\partial y_{i}}=\frac{q_{i} y_{i} k_{n}}{2 \rho_{i}}\left[I_{m-1}\left(k_{n} \rho_{i}\right)+I_{m+1}\left(k_{n} \rho_{i}\right)\right] \sin \left[m \theta_{i}\right] \sin \left[k_{n} z_{i}\right]+ \\
& \frac{q_{i} x_{i} m}{\rho_{i}^{2}} I_{m}\left(k_{n} \rho_{i}\right) \cos \left[m \theta_{i}\right] \sin \left[k_{n} z_{i}\right] \\
& \frac{\partial g_{1}}{\partial y_{i}}=\frac{q_{i} y_{i} k_{n}}{2 \rho_{i}}\left[I_{-1}\left(k_{n} \rho_{i}\right)+I_{1}\left(k_{n} \rho_{i}\right)\right] \cos \left[k_{n} z_{i}\right] \\
& \frac{\partial g_{2}}{\partial y_{i}}=\frac{q_{i} y_{i} k_{n}}{2 \rho_{i}}\left[I_{-1}\left(k_{n} \rho_{i}\right)+I_{1}\left(k_{n} \rho_{i}\right)\right] \sin \left[k_{n} z_{i}\right] \\
& \frac{\partial h_{1}}{\partial y_{i}}=\frac{q_{i} y_{i} m}{R \rho_{i}}\left(\frac{\rho_{i}}{R}\right)^{m-1} \cos \left[m \theta_{i}\right]-\frac{q_{i} x_{i} m}{\rho_{i}^{2}}\left(\frac{\rho_{i}}{R}\right)^{m} \sin \left[m \theta_{i}\right] \\
& \frac{\partial h_{2}}{\partial y_{i}}=\frac{q_{i} y_{i} m}{R \rho_{i}}\left(\frac{\rho_{i}}{R}\right)^{m-1} \sin \left[m \theta_{i}\right]+\frac{q_{i} x_{i} m}{\rho_{i}^{2}}\left(\frac{\rho_{i}}{R}\right)^{m} \cos \left[m \theta_{i}\right] \text {. }
\end{aligned}
$$


The $z$-derivatives are:

$$
\begin{gathered}
\frac{\partial f_{1}}{\partial z_{i}}=-q_{i} k_{n} I_{m}\left(k_{n} \rho_{i}\right) \cos \left[m \theta_{i}\right] \sin \left[k_{n} z_{i}\right] \\
\frac{\partial f_{1}}{\partial z_{i}}=q_{i} k_{n} I_{m}\left(k_{n} \rho_{i}\right) \cos \left[m \theta_{i}\right] \cos \left[k_{n} z_{i}\right] \\
\frac{\partial f_{3}}{\partial z_{i}}=-q_{i} k_{n} I_{m}\left(k_{n} \rho_{i}\right) \sin \left[m \theta_{i}\right] \sin \left[k_{n} z_{i}\right] \\
\frac{\partial f_{4}}{\partial z_{i}}=q_{i} k_{n} I_{m}\left(k_{n} \rho_{i}\right) \sin \left[m \theta_{i}\right] \cos \left[k_{n} z_{i}\right] \\
\frac{\partial g_{1}}{\partial z_{i}}=-q_{i} k_{n} I_{0}\left(k_{n} \rho_{i}\right) \sin \left[k_{n} z_{i}\right] \\
\frac{\partial g_{2}}{\partial z_{i}}=q_{i} k_{n} I_{0}\left(k_{n} \rho_{i}\right) \cos \left[k_{n} z_{i}\right] \\
\frac{\partial h_{1}}{\partial z_{i}}=\frac{\partial h_{2}}{\partial z_{i}}=0
\end{gathered}
$$

